\title{
Severe intellectual disability and autistic features associated with microduplication 2 q23.1
}

\author{
Brian HY Chung ${ }^{1}$, Sureni Mullegama ${ }^{2}$, Christian R Marshall ${ }^{3}$, Anath C Lionel ${ }^{3,4}$, Rosanna Weksberg ${ }^{1,4}$, \\ Lucie Dupuis ${ }^{1}$, Lauren Brick ${ }^{5}$, Chumei $\mathrm{Li}^{5}$, Stephen W Scherer ${ }^{3,4}$, Swaroop Aradhya ${ }^{6}$, D James Stavropoulos ${ }^{7,8}$, \\ Sarah H Elsea ${ }^{2,9}$ and Roberto Mendoza-Londono ${ }^{\star, 1}$
}

\begin{abstract}
We report on two patients with developmental delay, hypotonia, and autistic features associated with duplications of chromosome region 2q23.1-2q23.2 detected by chromosome microarray analysis. The duplications include one OMIM Morbid Map gene, MBD5, as well as seven known RefSeq genes (ACVR2A, ORC4L, EPC2, KIF5C, MIR1978, LYPD6B, and LYPD6). $M B D 5$ lies in the minimum area of overlap of the 2q23.1 microdeletion syndrome. This report provides the first detailed clinical examination of two individuals with a duplication of this region and suggests that brain development and cognitive function may be affected by an increased dosage of the genes involved.
\end{abstract}

European Journal of Human Genetics (2012) 20, 398-403; doi:10.1038/ejhg.2011.199; published online 16 November 2011

Keywords: 2q23.1 microduplication; MBD5 gene; CGH microarray; autism spectrum disorder

\section{INTRODUCTION}

Intellectual disability (ID) and developmental delay (DD) are common indications for referral to clinical geneticists. Developmental disorders are estimated to affect $1-3 \%$ of all children. ${ }^{1}$ In this patient population, the diagnostic yield of conventional karyotyping for chromosomal aberrations is $\sim 10 \% .^{2}$ Array comparative genomic hybridization (array CGH) overcomes the limited resolution of conventional cytogenetics and allows genome-wide scanning for submicroscopic chromosomal aberrations. ${ }^{3}$ Multiple studies of array $\mathrm{CGH}$ for patient cohorts with ID/DD and a normal karyotype by $\mathrm{G}$ banding have established an abnormality rate of $\sim 10-15 \%$. $^{2-5}$

Microdeletions of chromosome $2 \mathrm{q} 23.1$ are associated with a recently described syndrome characterized by $\mathrm{DD}$, language impairment, stereotypic repetitive movements, microcephaly, growth retardation, seizures, and ataxia, reminiscent of the Angelman or Rett syndromes. ${ }^{6-8}$ The deleted segments in the patients described to date are of variable size ranging from $250 \mathrm{~kb}$ to $5.5 \mathrm{Mb}$, with a common area of overlap including the MBD5 gene. ${ }^{8}$ Duplications of chromosome segments involved in microdeletion syndromes have been reported for several common conditions. A well-known example is the duplication of chromosome region $17 \mathrm{p} 12$ that causes CharcotMarie-Tooth disease type $1 \mathrm{~A}$ syndrome and the corresponding deletion that causes hereditary neuropathy with liability to pressure palsies. ${ }^{9}$ Other examples include duplications reciprocal to the recurrent deletions associated with Willams-Beuren syndrome, ${ }^{10}$ velo-cardio-facial syndrome, ${ }^{11}$ Smith-Magenis syndrome, ${ }^{12}$ neurofibromatosis I, ${ }^{13}$ Sotos syndrome, ${ }^{14}$ and Rett syndrome. ${ }^{15}$ Duplications of chromosome region 2q23.1 have not been described to date and the clinical consequences are currently not known. In this study, we report on two patients with microduplication of chromosome $2 \mathrm{q} 23.1-2 \mathrm{q} 23.2$ who presented with variable degrees of DD and autistic features and, in one case, aggressive behavior.

\section{CASE PRESENTATIONS}

Patient 1 was conceived naturally to a 22-year-old, nulliparous healthy mother and a 26-year-old father. There were no complications during pregnancy. The patient was delivered at 40 weeks gestation with a birth weight of $2630 \mathrm{~g}$ (third percentile). She remained in the hospital for 2 weeks because of poor feeding and hypotonia. A small apical muscular ventricular septal defect was confirmed by echocardiogram and later closed spontaneously. Her karyotype was normal 46,XX. Screenings for in utero infections by toxoplasma, rubella, cytomegalovirus, and herpes (TORCH) were negative. The proband was examined by a clinical geneticist at 2 weeks of age owing to hypotonia and subtle dysmorphic facial features. She had an outer canthal distance of $7 \mathrm{~cm}(>2 \mathrm{SD}$ ), inner canthal distance of $2.3 \mathrm{~cm}$ (mean to $+2 \mathrm{SD})$, and an interpupilary distance of $4.75 \mathrm{~cm}(0.25 \mathrm{~cm}>97 \mathrm{th}$ centile). The rest of her physical examination was unremarkable.

The patient was followed up in Genetics yearly until shortly before the age of 5 years. Her physical features remained unchanged. She had delay in all areas of development and attended an early intervention program for the first 2 years of life. She walked independently at 18 months and started climbing stairs at 4 years of age. She could run with an unsteady gait. Her first word was at age two and her vocabulary was limited. At 4 years and 8 months, she was diagnosed with global DD. She attended a school for children with special needs.

\footnotetext{
${ }^{1}$ Department of Pediatrics, Division of Clinical and Metabolic Genetics, Hospital for Sick Children, Toronto, Ontario, Canada; ${ }^{2}$ Department of Human and Molecular Genetics, Virginia Commonwealth University School of Medicine, Richmond, Virginia, USA; ${ }^{3}$ The Centre for Applied Genomics, Hospital for Sick Children, Toronto, Ontario, Canada; ${ }^{4}$ The McLaughlin Centre for Molecular Medicine, Department of Molecular Genetics, University of Toronto, Toronto, Ontario, Canada; ${ }^{5}$ Department of Pediatrics, Clinical Genetics Program, McMaster University Medical Center and McMaster Children's Hospital, Hamilton, Ontario, Canada; ${ }^{6}$ GeneDx, Gaithersburg, MD, USA; ${ }^{7}$ Department of Pediatric Laboratory Medicine, Cytogenetics Laboratory, Hospital for Sick Children, Toronto, Ontario, Canada; ${ }^{8}$ Department of Laboratory Medicine and Pathology, University of Toronto, Toronto, Ontario, Canada; ${ }^{9}$ Department of Pediatrics, Virginia Commonwealth University School of Medicine, Richmond, Virginia, USA

${ }^{*}$ Correspondence: Dr R Mendoza-Londono, Division of Clinical and Metabolic Genetics, Hospital for Sick Children and University of Toronto, Toronto, Ontario, Canada M5G 1 X8. Tel: 416-813-5340; Fax: 416-813-5345; E-mail: roberto.mendoza@sickkids.ca

Received 26 October 2010; revised 22 August 2011; accepted 27 September 2011; published online 16 November 2011
} 
An assessment at age of 8 years confirmed a diagnosis of autism. She had a total vocabulary of 20 words, but used them infrequently and inconsistently. She rarely made requests verbally. She did not point at pictures in a book or at objects. She made little eye contact. She would often hug strangers. She liked feeling other people's hands and faces and displayed some obsessive tendencies. On the Vineland Adaptive Behaviour Scales, her functional development was approximately at an age range of $1-2$ years.

On examination at 17 years of age, her head circumference was $53.5 \mathrm{~cm}$ (25th centile), height was $162.3 \mathrm{~cm}$ (50th centile), and weight was $46.7 \mathrm{~kg}$ (third-tenth centile; Figure 1). The anterior hairline was low lying. She had arched eyebrows and bilateral ptosis. Her nasal tip was bulbous. The philtrum appeared normal, her lips were prominent, and there was mild retrognathia. There was no limitation of joint movement. She had normal tone and muscle bulk. Her gait was wide based. Deep tendon reflexes were $2+$ bilaterally and plantar responses were flexor. Her hearing and vision were normal. She did not have any seizures. She gradually became more aggressive and emotionally unstable, and there were episodes of aggressive outbursts requiring hospitalization. From a functional perspective, she was able to feed herself but required help and supervision in other aspects of daily living. She had significant difficulties with communication.
A brain MRI at 8 years of age was normal. Fragile X testing was negative. Methylation studies and fluorescence in situ hybridization (FISH) analysis for Prader-Willi/Angelman syndromes were normal. Metabolic workup including plasma amino acids, lactate, thyroid stimulating hormone, blood gases, glucose, plasma total/free carnitine and acylcarnitine profile, transferrin isoelectric-focusing, urine organic acids, urine oligosaacharides, and mucopolysaccharides were all normal.

The patient is the only child born to healthy non-consanguineous parents. Her father was of Scottish descent and has no further children. Her mother was of African-Caribbean and British descent. She has four other children from two different partners. One of our patient's half-sisters had been diagnosed with mild DD. The rest of the family history was unremarkable.

Patient 2 was an 8-year-old male of Caucasian descent who was referred for genetics evaluation because of DD and autistic features. He was born to a 32-year-old mother after a pregnancy complicated by mild vaginal spotting. He was at 40 weeks gestation with no complications. His birth weight was $3203 \mathrm{~g}$ and birth length $53 \mathrm{~cm}$. $\mathrm{He}$ had poor neonatal feeding, hypotonia, and experienced gastroesophageal reflux with recurrent vomiting in the first year of life. He was diagnosed with strabismus and astigmatism.
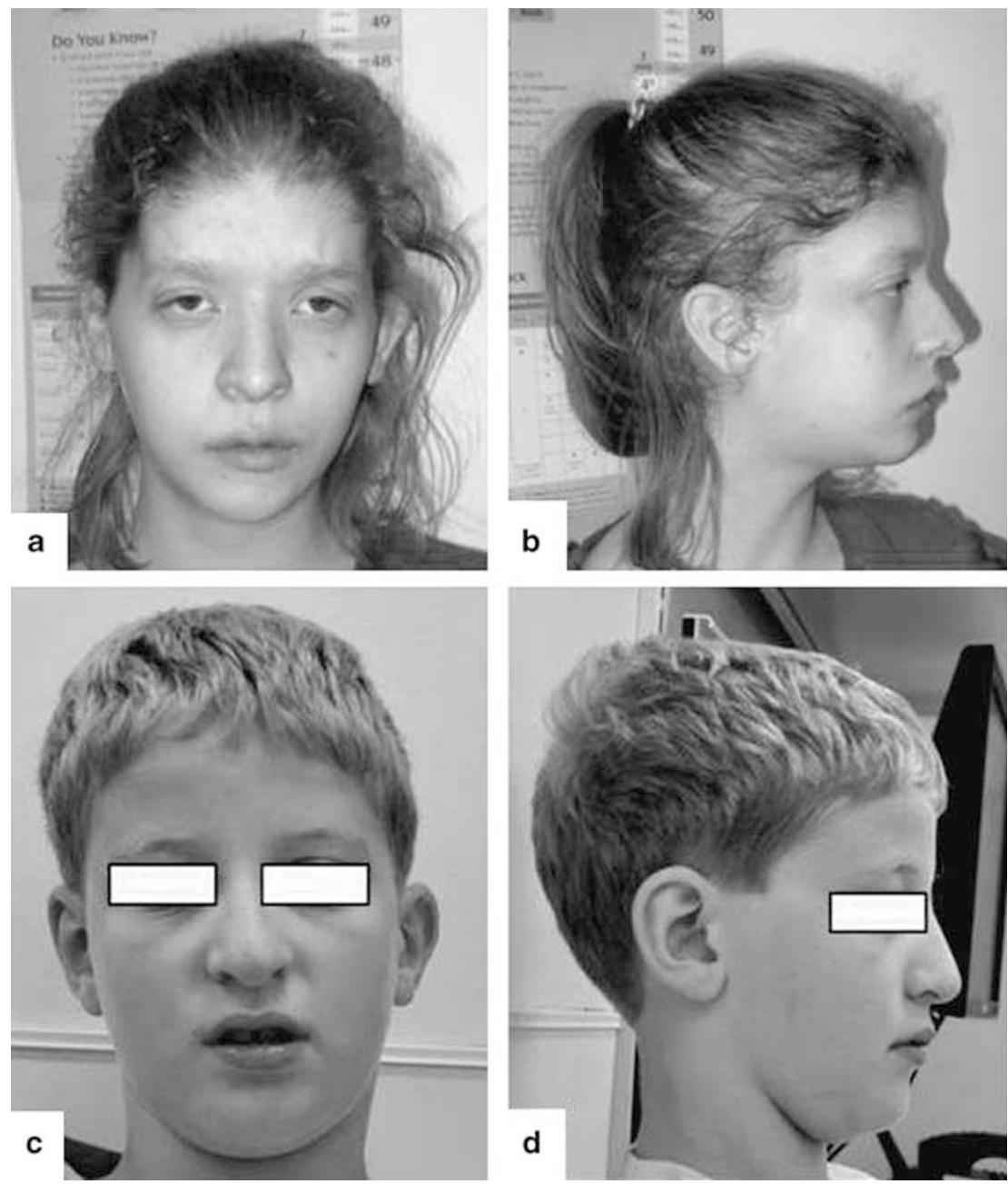

Figure 1 Facial dysmorphology in patients with duplication in 2q23.1. Clinical photographs of Patient 1 (a, b) showing arched eyebrows, bilateral ptosis, bulbous nasal tip, prominent lips, and mild retrognathia. Clinical photographs of Patient 2 (c, d) revealing subtle facial asymmetry with the right side slightly smaller than the left side, a tubular nose with prominent nasal tip, and somewhat prominent lips. 
The patient had global DD. He sat unsupported at 10 months, crawled at 22 months, but was unable to walk or talk until the age of 2 years. He was toilet trained at 4.5 years of age. At 8 years of age, he was attending a regular grade 3 class with an individualized education plan in place for mathmathematics and language. He was performing at a senior kindergarten level for reading and writing. He had fine motor delay, mild persistent hypotonia, poor coordination, and widebased gait. His personality was described as 'friendly and social'.

On physical exam at 8 years of age, his growth parameters were all above the 98th centile, with head circumference of $55.5 \mathrm{~cm}$, height of $144.5 \mathrm{~cm}$, and weight of $39 \mathrm{~kg}$. Craniofacial examination revealed a subtle facial asymmetry, with right hemifacial microsomia, strabismus, high-arched palate, and dental crowding (Figure 1). His nose was long and tubular and had a prominent tip. He had a $5 \times 2 \mathrm{~cm}$ café-au-lait spot on the right thorax. Extremities showed bilateral fifth finger clinodactyly, a hint of fetal fat pads on the two to fourth fingers bilaterally, and large great toes.

Investigations done as part of his work-up included G-banded karyotype at a 550-band resolution that showed a normal male chromosome complement. Subtelomeric FISH analysis and FISH for 16 p13.3 to rule out Rubinstein-Taybi syndrome were normal. Fragile $\mathrm{X}$ molecular testing, thyroid function testing, and metabolic screening including urine organic acids, urine pterins, galactosemia screen, and liver function tests were normal. Bone age was carried out because of his macrosomia, and persistent large size showed bone maturation concordant with his chronological age. Electroencephalogram was performed to rule out seizure activity and had normal tracing. Head CT confirmed skull asymmetry but did not show evidence of craniosynostosis. MRI of the brain at 2 years of age showed mild right-sided hemiatrophy.

The patient was the second child to non-consanguineous parents of German and Polish descent. He has one older brother who is healthy. The family history was positive for learning difficulties in the mother, two of her brothers, and her father. Otherwise, family history was unremarkable.

\section{MATERIALS AND METHODS \\ Cell lines}

Lymphoblastoid cell lines (human lymphocytes transformed with Epstein-Barr virus) from patient 1 and unaffected controls were cultured according to the standard methods.

\section{Array CGH}

Array CGH for patient 1 was performed using the oligonucleotide $4 \times 44 \mathrm{~K}$ microarray platform (Agilent Technologies, Santa Clara, CA, USA) with a custom design described previously. ${ }^{16}$ Patient and pooled same-sex reference DNA (Promega, Madison, WI, USA) were labeled with Cy3-dCTP and Cy5dCTP, respectively, and hybridized to the array platform, as recommended by the manufacturer's protocol (Agilent Technologies). The arrays were scanned using the Agilent G2505B microarray scanner. Data analysis was performed using DNA Analytics version 4.0 with the ADM-1 algorithm set at a threshold of 6.7 and a minimum of four contiguous probes (Agilent Technologies). FISH analysis was performed using standard protocols with an FITC-labeled bacterial artificial chromosome probe (RP11-375H16) obtained from The Centre for Applied Genomics (Toronto, Canada). Array CGH screening of patient 2 was performed using a whole-genome microarray that contained 180000 oligonucleotide probes spaced $35 \mathrm{~kb}$ apart throughout the genome and at 5-20 kb in over 150 clinically significant regions (GeneDx, Gaithersburg, MD, USA). The array hybridization was performed as described above, with some minor differences.

\section{RNA extraction}

RNA was extracted from whole blood $(8 \mathrm{ml})$ from patient 1 and a healthy control using the standard TRIzol protocol (Invitrogen Canada Inc.,
Burlington, Ontario, Canada). The concentration and purity of RNA were measured at absorbances of 260 and $280 \mathrm{~nm}$. RNA was stored at $-80^{\circ} \mathrm{C}$ until use.

\section{Quantitative real-time (qRT)-PCR}

First-strand cDNA synthesis using SuperScript II RT (Invitrogen) was performed with $5 \mu \mathrm{g}$ of total RNA from patient 1 and a healthy control using Oligo(dT)12-18, and RNA degradation with 2.5. U RNase H (Applied Biosystems Inc., Foster City, CA, USA). For qRT-PCR, predesigned assays on Demand Gene Expression Products (Fermentus, Glen Burnie, MD, USA) Taqman MGB probes for MBD5, EPC2, and GAPDH were used (Applied Biosystems Inc.). GAPDH was used as the endogenous control. All samples of cDNA were run in triplicate in $10 \mu \mathrm{l}$ reaction volumes. Taqman Universal PCR Master Mix (Applied Biosystems Inc.), probe, and deionized water were mixed in a fixed ratio, and $8 \mu \mathrm{l}$ was added to each well. Diluted cDNA (1:2) was then added to each well. PCR conditions were the standard 7500 Run mode of the ABI Prism 7900 HT Sequence Detection System (Applied Biosystems Inc.). The cycle threshold was determined during the geometric phase of the PCR amplification plots, as recommended by the manufacturer. Relative differences in transcript levels were quantified using the $\Delta \Delta \mathrm{Ct}$ method. Data were analyzed using the 7500 Fast Systems SDS Software (Applied Biosystems Inc.). Patient 2 did not contribute a sample for RNA extraction.

\section{RESULTS}

\section{Array CGH}

Patient 1: A customized 44K oligonucleotide array identified a duplication of chromosome region 2q23. 1-2q23.2, represented by 24 oligonucleotide probes from position $148691798-150343042042$ (NCBI Build 3737/hg19). No other clinically significant CNVs were identified by this analysis. The estimated size of the copy number gain was $1.64 \mathrm{Mb}$, with adjacent oligonucleotide probes at positions 148616673 and 150420742 showing normal copy number (Figure 2a). FISH analysis using the RP11-375H16 probe was consistent with a duplication of this region (Figure 2c). FISH analysis of the maternal sample and a sample from the patient's half sister with DD showed normal results (data not shown). A paternal sample could not be obtained for follow-up studies. This genomic region contains seven known RefSeq genes including ORC4L (OMIM 603056), EPC2 (OMIM 611000), KIF5C (OMIM 604593), MIR1978, LYPD6B, LYPD6, and OMIM Morbid Map gene MBD5 (OMIM 611472).

Patient 2: Array CGH identified a de novo duplication of chromosome region 2q22.3q23.2 from position 148 186210-150 188492 bp (NCBI Build 37/hg19) as the maximum interval and from position 148 616 673-150 420742 as the minimal interval (Figure 2b). No other CNV's considered clinically significant were identified by this analysis. The estimated size of the duplication is $2 \mathrm{Mb}$. Analysis of parental samples by array CGH with a whole-genome $60 \mathrm{~K}$ array showed normal copy number for the 2q22.3-2q23.2 region (data not shown), indicating that the duplication in the proband was a de novo occurrence. This genomic region contains seven known RefSeq genes including ACVR2A (OMIM 102581), ORC4L (OMIM 603056), EPC2 (OMIM 611000), KIF5C (OMIM 604593), LYPD6B, LYPD6 (OMIM 613359), and OMIM Morbid Map gene MBD5 (OMIM 611472).

\section{Control populations}

Deletions within this genomic region, including the MBD5 gene, have previously been reported to be associated with neurodevelopmental abnormalities. ${ }^{2,5-8}$ A search of the Database of Genomic Variants (DGV) indicated a copy number gain detected in one individual from one study of 776 control samples from individuals in Northern Germany collected as part of the PopGen project. ${ }^{17}$ The Children's Hospital of Philadelphia structural variation project did not find duplications of this region in 2026 presumably healthy individuals, 


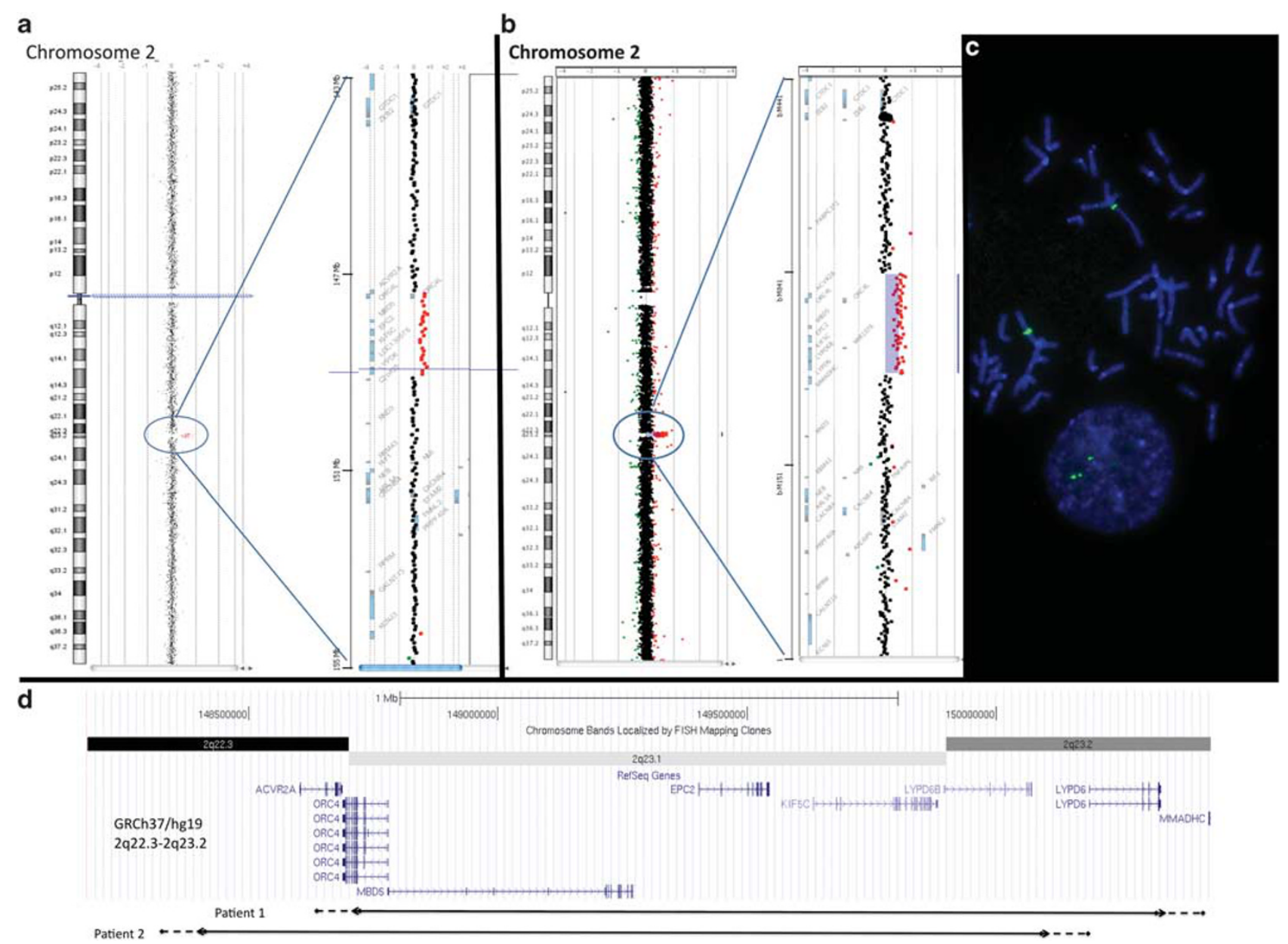

Figure 2 Array CGH and FISH analysis. Molecular cytogenetic investigations in patients 1 and 2 identified similar duplications within the long arm of chromosome 2. The estimated size of the duplications are 1.634 and $2 \mathrm{Mb}$, respectively, and both involve seven RefSeq genes including MBD5 and EPC2. (a, b) Chromosome and Gene views of the duplications obtained from DNA Analytics 4.0 (Agilent Technologies). (c) FISH analysis of Iymphocytes from patient 1 using a FITC-labeled RP11-375H16 bacterial artificial chromosome probe (green) on DAPI-stained nuclei (blue) is consistent with a duplication. (d) Gene content displayed on the UCSC Genome Browser (NCBI 37/hg19).

confirming that it was rarely observed in presumed healthy populations. ${ }^{18}$ We examined this locus for CNVs in high-resolution microarray data that had been analyzed by us in 8427 control individuals. ${ }^{19}$ The control data set included Affymetrix SNP 6.0 (Affymetrix, Santa Clara, CA, USA) data from 1123 controls recruited from northern Germany as a part of the PopGen project, ${ }^{20} 1234$ healthy controls recruited from the province of Ontario (Canada) for a coronary artery disease study by the Ottawa Heart Institute $(\mathrm{OHI}),{ }^{21}$ and 4783 British controls from the Wellcome Trust Case Control Consortium (WTCCC), ${ }^{16}$ and Illumina Infinium $1 \mathrm{M}$ (Illumina Inc., San Diego, CA, USA) data from 1287 controls recruited by the Study of Addiction: Genetics and Environment (SAGE) consortium. ${ }^{22}$ No CNVs were detected in these controls of comparable size and extent as the 2q23.1 duplications in these two patients. There were six small intragenic variants of sizes $<100 \mathrm{~kb}$, which did not overlap with any of the genes in the region.

\section{Real-time qRT-PCR}

Gene expression studies were conducted for two genes in the area of duplication with freshly isolated lymphocytes from patient 1 . Figure 3 shows expression of MBD5 and EPC2 relative to GAPDH expression and two different control samples, with relative expression values based on the $\Delta \Delta \mathrm{Ct}$ value with control cells set to 1 . Average values for all combined experiments, conducted in triplicate, are shown $(n=3-4)$. The data showed that both MBD5 and EPC2 were overexpressed in this individual, supporting a role for these genes in the phenotype observed.

\section{DISCUSSION}

In previously reported cases of microdeletion 2q23. 1 syndrome, haploinsufficiency of genes, including MBD5 and EPC2, within the overlapping deletion region is thought to be causative of the phenotype. These two genes are found in the common deletion region in the five cases summarized by Jaillard et al. ${ }^{7}$ Williams et al ${ }^{23}$ have presented evidence of a $50 \%$ reduction in expression of these genes in lymphoblasts/lymphocytes of two patients with a 2q23.1 deletion. The case reported by Wagenstaller et al had a small $200-\mathrm{kb}$ deletion including only the MBD5 gene. This patient reportedly had ID, motor delay, and febrile seizures with no dysmorphic features. These observations suggest that deletion of the MBD5 gene could be sufficient to cause ID. The duplications in the two cases reported here contained seven known RefSeq genes, including MBD5 and EPC2. The MBD5 gene is a member of the methyl-binding (MB) gene family, and the protein contains an $\mathrm{MB}$ domain, which is shared by MECP2 and other MBD 

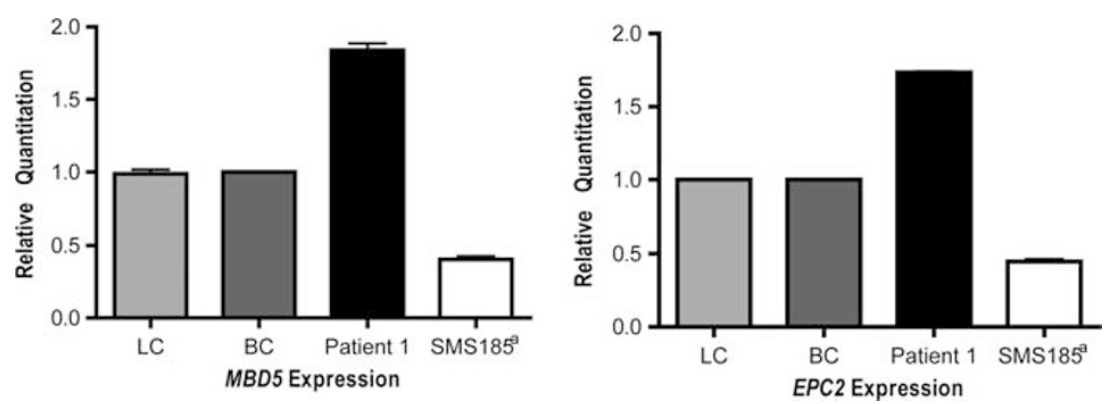

Figure 3 Elevated expression of MBD5 and EPC2 in 2q23.1 duplication syndrome. qRT-PCR mRNA expression analysis of MBD5 (left) and EPC2 (right) in lymphoblastoid cell lines or fresh white blood cells from patient 1 with 2q23.1 duplication. Fresh white blood cells or lymphoblastoid cell lines from unaffected (absence of alteration of MBD5 or EPC2) subjects were used as normal controls. Results were normalized to GAPDH expression. Relative expression values are based on the $\Delta \Delta \mathrm{Ct}$ value. Expression of all controls was normalized to 1 . Each bar represents the mean ( $\pm \mathrm{SEM}$ ) of values from three to five independent experiments. Data show increased MBD5 (left) and EPC2 (right) expression in Patient $1 .{ }^{a} \mathrm{~A}$ previously published case, SMS185 (Williams et $a^{23}$ ), revealed a reduction of MBD5 and EPC2 due to haploinsufficiency (left).

proteins. ${ }^{24}$ MECP2 is a dosage-sensitive gene, and both deletions and duplications of this gene are associated with ID and other neurodevelopmental features. ${ }^{15}$ It is not yet known whether a copy number gain of MBD5 and surrounding genes in chromosome region $2 \mathrm{q} 23.1$ is pathogenic. Duplications of chromosome region 2q23.1 are very rare events in the general population. Our review of extensive highresolution control data sets did not reveal a similar duplication in over 8427 individuals. A review of the DGV revealed one report of a copy number gain in a single individual. The copy number data were not experimentally validated by an independent method. Obviously, a single report on the DGV has the potential of representing a falsepositive call. Furthermore, assuming that the duplication represented on the DGV is a true call, this copy number change is indeed rare in healthy control populations given that it was only found once in this large sample of individuals. A duplication of 2q23.1, including the MBD5 gene, was identified by Pinto et al ${ }^{19}$ in a male patient with autism, mild ID, relative macrocephaly, and dysmorphic features. The authors interpreted their data to suggest that this chromosome change was not responsible for the phenotype, based on the fact that the duplication was inherited from an unaffected mother, and that the patient had symptoms different from those seen in the 2q23.1 microdeletion syndrome. However, it has been shown that the clinical presentations due to microduplications of a given genomic segment can be significantly different from those seen in the corresponding microdeletion syndrome. ${ }^{10-15}$ Furthermore, microduplications that are responsible for clinical phenotypes may be inherited from apparently unaffected carrier parents. ${ }^{25-27}$ Repnikova et al ${ }^{28}$ have reported a patient with a $600-\mathrm{kb}$ duplication in chromosome $2 \mathrm{q} 23.1$ that included MBD5 and EPC2. This patient presented an Angelman-like phenotype with mild ID and seizures. In light of these findings and those reported in our patients who carry a de novo duplication in patient 2 and a duplication of unknown origin in patient 1 , the potential pathogenicity of this duplication could be reevaluated. The DECIPHER database has two duplications that involve MBD5 in individuals reported to have ataxia, DD, and tall stature (DECIPHER ID 251363), or deafness (DECIPHER ID 248386).

A reciprocal phenotype between deletion and duplication cases has been observed in some genomic disorders. ${ }^{10,25}$ Understanding the clinical significance of duplications in chromosome regions known to be pathogenic because of haploinsufficiency is often difficult as copy number gains can confer a milder phenotype and are often found to be inherited. ${ }^{26,27}$ There are also a number of genomic regions, such as $16 \mathrm{p} 13.11$, for which copy number loss is associated with neurodevelopmental abnormalities, but evidence of pathogenicity for the reciprocal duplication is currently lacking. ${ }^{25}$ At least 15 cases with a 2q23.1 deletion have been reported, ${ }^{6-8}$ and clinical features in more than half of the patients included DD, language impairment, stereotypic repetitive movement, microcephaly, growth retardation, seizures, ataxia, and minor hand/food anomalies. Language delay and stereotypic behaviors were found in the majority of the reported patients, and two patients were suspected to have autism. van Bon et $a l^{8}$ have commented that they were unable to delineate a recognizable pattern of facial dysmorphism in patients affected with the 2q23.1 deletion. In our study, both patients had ID, autistic features, and patient 1 displayed aggressive behavior during adolescence even though she did not display this phenotype as a child. Neither patient had the seizures nor growth issues observed in the patients with the microdeletion.

Our findings raise the possibility that rare duplications of chromosome region 2q23.q23.2 that includes MBD5 may be associated with DD and autistic features. Our data serve as an initial observation to prime other clinical genetics and control reports necessary to make a definitive causal association.

\section{CONFLICT OF INTEREST}

The authors declare no conflict of interest.

\section{ACKNOWLEDGEMENTS}

We thank the families for participating in the study, as well as The Centre for Applied Genomics (http://www.tcag.ca). We also thank A Fiebig, A Franke, and S Schreiber at POPGEN (University of Kiel, Kiel, Germany), A Stewart, R McPherson, and R Roberts of the University of Ottawa Heart Institute (University of Ottawa, Ottawa, Canada), the Wellcome Trust Case Control Consortium (WTCCC), the DECIPHER Consortium, and the Study of Addiction: Genetics and Environment (SAGE) consortium for generously providing population control microarray data. SWS is supported by The Centre for Applied Genomics (http://www.tcag.ca), Genome Canada and the Ontario Genomics Institute, the Canadian Institutes for Health Research (CIHR), the Canadian Institute for Advanced Research (CIFAR), the McLaughlin Centre, the Canada Foundation for Innovation, the Ontario Ministry of Research and Innovation and the Hospital for Sick Children Foundation. SWS holds the GlaxoSmithKline-CIHR Chair in Genetics and Genomics at the University of Toronto and the Hospital for Sick Children. This study was supported, in part, by the Fondation Jérome Lejeune (SHE).

\footnotetext{
1 Shevell M, Ashwal S, Donley D et al: Practice parameter: evaluation of the child with global developmental delay: report of the Quality Standards Subcommittee of the American Academy of Neurology and The Practice Committee of the Child Neurology Society. Neurology 2003; 60: 367-380.
} 
2 de Vries BB, Pfundt R, Leisink $M$ et al: Diagnostic genome profiling in mental retardation. Am J Hum Genet 2005; 77: 606-616.

3 Fan YS, Jayakar $\mathrm{P}$, Zhu $\mathrm{H}$ et al: Detection of pathogenic gene copy number variations in patients with mental retardation by genomewide oligonucleotide array comparative genomic hybridization. Hum Mutat 2007; 28: 1124-1132.

4 Friedman JM, Baross A, Delaney AD et al: Oligonucleotide microarray analysis of genomic imbalance in children with mental retardation. Am J Hum Genet 2006; 79 500-513.

5 Wagenstaller J, Spranger S, Lorenz-Depiereux B et al: Copy-number variations measured by single-nucleotide-polymorphism oligonucleotide arrays in patients with mental retardation. Am J Hum Genet 2007; 81: 768-779.

6 Chung BH, Stavropoulos J, Marshall CR, Weksberg R, Scherer SW, Yoon G: 2q23 de novo microdeletion involving the MBD5 gene in a patient with developmental delay, postnatal microcephaly and distinct facial features. Am J Med Genet A 2011; 155A: 424-429.

7 Jaillard S, Dubourg C, Gerard-Blanluet M et al: 2q23.1 microdeletion identified by array comparative genomic hybridisation: an emerging phenotype with Angelman-like features? J Med Genet 2009; 46: 847-855.

8 van Bon BW, Koolen DA, Brueton L et al: The 2q23.1 microdeletion syndrome: clinical and behavioural phenotype. Eur J Hum Genet 2010; 18: 163-170.

9 Chance PF, Abbas N, Lensch MW et al: Two autosomal dominant neuropathies result from reciprocal DNA duplication/deletion of a region on chromosome 17. Hum Mol Genet 1994; 3: 223-228.

10 Somerville MJ, Mervis CB, Young EJ et al: Severe expressive-language delay related to duplication of the Williams-Beuren locus. N Engl J Med 2005; 353: 1694-1701.

11 Ensenauer RE, Adeyinka A, Flynn HC et al: Microduplication 22q11.2, an emerging syndrome: clinical, cytogenetic, and molecular analysis of thirteen patients. Am J Hum Genet 2003; 73: 1027-1040.

12 Potocki L, Chen KS, Park SS et al: Molecular mechanism for duplication 17p11.2- the homologous recombination reciprocal of the Smith-Magenis microdeletion. Nat Genet 2000; 24: 84-87.

13 Grisart B, Rack K, Vidrequin S et al: NF1 microduplication first clinical report: association with mild mental retardation, early onset of baldness and dental enamel hypoplasia? Eur J Hum Genet 2008; 16: 305-311.

14 Franco LM, de Ravel T, Graham BH et al: A syndrome of short stature, microcephaly and speech delay is associated with duplications reciprocal to the common Sotos syndrome deletion. Eur J Hum Genet 2010; 18: 258-261.
15 Van Esch H, Bauters M, Ignatius J et al: Duplication of the MECP2 region is a frequent cause of severe mental retardation and progressive neurological symptoms in males. Am J Hum Genet 2005; 77: 442-453.

16 Craddock N, Hurles ME, Cardin N et al: Genome-wide association study of CNVs in 16000 cases of eight common diseases and 3000 shared controls. Nature 2010; 464: 713-720.

17 Pinto D, Marshall C, Feuk L, Scherer SW: Copy-number variation in control population cohorts. Hum Mol Genet 2007; 16 (Spec no. 2): R168-R173.

18 Shaikh TH, Gai X, Perin JC et al: High-resolution mapping and analysis of copy number variations in the human genome: a data resource for clinical and research applications. Genome Res 2009; 19: 1682-1690.

19 Pinto D, Pagnamenta AT, Klei L et al: Functional impact of global rare copy number variation in autism spectrum disorders. Nature 2010; 466: 368-372.

20 Krawczak M, Nikolaus S, von Eberstein H, Croucher PJ, El Mokhtari NE, Schreiber S: PopGen: population-based recruitment of patients and controls for the analysis of complex genotype-phenotype relationships. Community Genet 2006; 9: 55-61.

21 Stewart AF, Dandona S, Chen L et al: Kinesin family member 6 variant Trp719Arg does not associate with angiographically defined coronary artery disease in the Ottawa Heart Genomics Study. J Am Coll Cardiol 2009; 53: 1471-1472.

22 Bierut LJ, Agrawal A, Bucholz KK et al: A genome-wide association study of alcohol dependence. Proc Natl Acad Sci USA 2010; 107: 5082-5087.

23 Williams SR, Mullegama SV, Rosenfeld JA et al: Haploinsufficiency of MBD5 associated with a syndrome involving microcephaly, intellectual disabilities, severe speech impairment, and seizures. Eur J Hum Genet 2010; 18: 436-441.

24 Turner H, MacDonald F, Warburton S, Latif F, Webb T: Developmental delay and the methyl binding genes. J Med Genet 2003; 40: E13.

25 Brunetti-Pierri N, Berg JS, Scaglia F et al: Recurrent reciprocal 1q21.1 deletions and duplications associated with microcephaly or macrocephaly and developmental and behavioral abnormalities. Nat Genet 2008; 40: 1466-1471.

26 Stankiewicz P, Pursley AN, Cheung SW: Challenges in clinical interpretation of microduplications detected by array CGH analysis. Am J Med Genet A 2010; 152A: 1089-1100.

27 Hannes FD, Sharp AJ, Mefford HC et al: Recurrent reciprocal deletions and duplications of 16p13.11: the deletion is a risk factor for MR/MCA while the duplication may be a rare benign variant. J Med Genet 2009; 46: 223-232.

28 Repnikova EA, Bailes A, McKinney A et al: Microdeletions and Microduplications of 2q23.1 involving MBD5 gene. Abstract \#829, 60th Annual Meeting of the American Society of Human Genetics. Washington DC, USA, November 42010. 